\title{
Veterinarians and Wildlife Biologists Should Join Forces to End Inhumane Mammal Trapping Technology
}

\author{
Gilbert Proulx \\ Alpha Wildlife Research \& Management Ltd., 229 Lilac Terrace, Sherwood Park, Alberta, T8H 1W3, Canada \\ *Corresponding author's Email: gproulx@alphawildlife.ca; (iDORCiD: 0000-0002-6980-8927
}

\begin{abstract}
Current mammal trapping standards uphold the use of inhumane trapping technology. For example, killing neck snares for the capture of canids, and rotating-jaw traps, and steel-jawed leghold traps for procyonids and mustelids, are being used by trappers despite decades of research showing that they are inhumane, and cause serious injuries and distress in captured animals. Many wildlife biologists unsuccessfully raised concerns about inhumane mammal trappings. This short communication stresses the need for veterinarians and wildlife biologists to work together to improve the fate of mammals captured in killing or restraining traps, and modify mammal trapping standards on the basis of animal welfare science.
\end{abstract}

Keywords: Humaneness, Mammal trapping, Traps, Trapping standards

\section{INTRODUCTION}

Trapping standards developed by the International Organization for Standardization (ISO) in the 1990s, the Agreement on International Humane trapping Standards (AIHTS) in 1997, and the USA Best Management Practices (White et al., 2021) are industrial standards to save the fur industry from trade bans (Proulx et al., 2020). These are sub-standard which do not meet, and have never met, state-of-the-art trapping technology and protocols associated with animal welfare (Proulx et al., 2020).

Red foxes (Vulpes vulpes), coyotes (Canis latrans), and grey wolves (Canis lupus) are snared annually in the fur trade or predator control programs in Canada and the United States (Iossa et al., 2007). However, nearly 40 years ago, Canadian researchers assessed the "humaneness" of manual killing neck snares, where the animal provides the energy necessary to tighten the noose and strangle itself, to quickly render red foxes irreversibly unconscious. They found that they could not cut off the airflow when the snare was held tight around the trachea (FPCHT, 1981). Less than a decade later, Proulx and Barrett (1990) showed that red foxes captured in power killing neck snares, where springs provide the energy to tighten the noose on an animal's neck, did not quickly lose consciousness. Finally, Proulx (2018) monitored a working trapline with remote video cameras and showed that a coyote and a wolf lost consciousness 14 hours 16 minutes, and 3 hours 39 minutes, respectively, after being captured in a manual killing neck snare. In both cases, animals struggled intensively and frequently, cut their tongue and gums, damaged their teeth, bled, and suffered from extreme swelling of the neck and head.

Killing neck snares are only examples of traps that are allowed in North America. Despite extensive research showing that rotating jaw-traps do not have the ability to quickly kill northern raccoons (Procyon lotor) (Proulx and Drescher, 1994), these traps have been certified (e.g., the Fur Institute of Canada, 2020) or accepted as Best Management Practices (AFWA, 2014). Likewise, steel-jawed leghold traps that seriously injure animals and cause them to selfmutilate (Proulx et al., 1993; White et al., 2021) are still being used in the United States.

\section{Inadequate Mammal Trapping Standards}

It is puzzling to see that traps that have been repeatedly found unacceptable for decades are still being used to capture mammals. Also, in most countries, animal anti-cruelty legislation is blatantly inadequate for wildlife. In the past, a handful of scientists have expressed opinions against inadequate mammal trapping standards and the use of trapping devices causing unacceptable pain and suffering (Iossa et al., 2007; Proulx and Rodtka, 2017; Proulx et al., 2020). Some environmental organizations have raised concerns but most of them are readily dismissed by government agencies (White et al., 2021).

In an effort to reform mammal trapping technology, and minimize harm to animals, veterinarians should join forces with wildlife biologists (Cattet, 2013). Wildlife biologists are responsible for the professional conservation and management of wildlife species and populations, and the wellbeing of individuals (Paquet and Darimont, 2010). The list 
of injuries associated with trapping devices that are technologically outdated suffices to illustrate that animal welfare groups' concerns are neither exaggerated nor overemotional. When wildlife biologists and veterinarians remain passive and do not stand against cruelty in mammal trapping, they fail to act as professionals dedicated to the sound management of wildlife populations and the wellbeing of animals.

\section{CONCLUSION}

Inadequate mammal trapping standards uphold the use of inhumane trapping technology. Both veterinarians and wildlife biologists should take the lead in examining and modifying mammal trapping standards on the basis of animal welfare science.

\section{DECLARATION}

\section{Acknowledgments}

I thank Dr. Daryoush Babazadeh and Ali Sadeghi for their comments on an earlier version of this manuscript.

\section{Competing interests}

I declare no conflict of interest.

\section{Consent to publish}

I agree with the publication of this paper.

\section{REFERENCES}

Association of Fish and Wildlife Agencies (AFWA) (2014). Best management practices for trapping raccoons in the United States. Mimeograph. Available at: https://www.dec.ny.gov/docs/wildlife_pdf/trapbmpsraccoon.pdf

Cattet MRL (2013). Falling through the cracks: Shortcomings in the collaboration between biologists and veterinarians and their consequences for wildlife. Institute for Laboratory Animal Research, 54: 33-40. DOI: https://www.doi.org/10.1093/ilar/ilt010

Federal Provincial Committee for Humane Trapping (FPCHT) (1981). Report of the federal provincial committee for humane trapping. Federal-Provincial Wildlife Conference, Ottawa, Ontario, Canada.

Iossa G, Soulsbury CD, and Harris S (2007). Mammal trapping: A review of animal welfare standards of killing and restraining traps. Animal Welfare, 16: 1-18. Available at: https://citeseerx.ist.psu.edu/viewdoc/download?doi=10.1.1.973.5675\&rep=rep1\&type=pdf

Paquet P, and Darimont CT (2010). Wildlife conservation and animal welfare: Two sides of the same coin?. Animal Welfare, 19: 177190. Available at: http://www.raincoast.org/wp-content/uploads/Paquet-and-Darimont-2010-Wildlife-Conservation-AnimalWelfare.pdf

Proulx G (2018). Intolerable cruelty - The truth behind killing neck snares and strychnine. Alpha Wildlife Publications, Sherwood Park, Canada, p. 96. Available at: https://cwbm.ca/product/intolerable-cruelty-the-truth-behind-killing-neck-snares-and$\underline{\text { strychninel }}$

Proulx G, and Barrett MW (1990). Assessment of power snares to effectively kill red fox. Wildlife Society Bulletin, 18: 27-30. Available at: https://alphawildlife.ca/wp-content/uploads/2015/03/25-1990-snare-red-fox.pdf

Proulx G, and Drescher RK (1994). Assessment of rotating-jaw traps to humanely kill raccoons. Journal of Wildlife Diseases, 30: 335-339. DOI: https://www.doi.org/10.7589/0090-3558-30.3.335

Proulx G, and Rodtka D (2017). Steel-jawed leghold traps and killing neck snares: Similar injuries command a change to agreement on international humane trapping standards. Journal of Applied Animal Welfare Science, 20: 198-203. DOI: https://www.doi.org/10.1080/10888705.2017.1286989

Proulx G, Cattet M, Serfass TL, and Baker SE (2020). Updating the AIHTS trapping standards to improve animal welfare and capture efficiency and selectivity. Animals, 10: 1262. DOI: https://www.doi.org/10.3390/ANI10081262

Proulx G, Onderka DK, Kolenosky AJ, Cole PJ, Drescher RK, and Badry MJ (1993). Injuries and behavior of raccoons (Procyon lotor) captured in the Soft Catch ${ }^{\mathrm{TM}}$ and the $\mathrm{EGG}^{\mathrm{TM}}$ traps in simulated natural environments. Journal of Wildlife Diseases, 29: 447-452. Available at: file:///C:/Users/Owner/Downloads/0090-3558-29.3.447.pdf

White HB, Boggess EK, Brown CL, Butfiloski JW, Decker TA, Erb JD, Fall MW, Hamilton DA, Hiller TL, Hubert GF Jr et al. (2021). Best management practices for trapping furbearers in the United States. Wildlife Monographs, 207: 3-59. DOI: https://doi.org/10.1002/wmon.1057 